\title{
Heat Shock Protein Grp78/BiP/HspA5 Binds Directly to TDP-43 and Mitigates Toxicity Associated with Neurodegenerative Disease Pathology
}

Liberty François-Moutal

University of Arizona

David Scott

University of Arizona

Andrew Ambrose

University of Arizona

Christopher Zerio

University of Arizona

Kumara Dissanayake

University of Dundee

Danielle May

Sanford Research

Jacob Carlson

University of Arizona

Edward Barbieri

University of Pennsylvania

Aubin Moutal

University of Arizona

Kyle Roux

Sanford Research

James Shorter

University of Pennsylvania

Rajesh Khanna

University of Arizona

\section{Sami Barmada}

University of Michigan-Ann Arbor

Leeanne McGurk

University of Dundee

May Khanna ( $\square$ maykhanna@email.arizona.edu )

University of Arizona 


\section{Research Article}

Keywords: TDP43, Hsp70, HspA5, BiP, GRP78, binding interface, ALS, chaperone

Posted Date: December 8th, 2021

DOI: https://doi.org/10.21203/rs.3.rs-1090289/v1

License: (c) (i) This work is licensed under a Creative Commons Attribution 4.0 International License. Read Full License 
Heat shock protein Grp78/BiP/HspA5 binds directly to TDP-43 and mitigates toxicity associated with neurodegenerative disease pathology

Liberty François-Moutal ${ }^{1,2}$, David Donald Scott ${ }^{1,2}$, Andrew J. Ambrose ${ }^{5}$, Christopher J. Zerio ${ }^{5}$, Kumara Dissanayake ${ }^{3}$, Danielle G. May ${ }^{4}$, Jacob M. Carlson ${ }^{1,2}$, Edward Barbieri ${ }^{6}$, Aubin Moutal ${ }^{1,2}$, Kyle J. Roux ${ }^{4,7}$, James Shorter ${ }^{6}$, Rajesh Khanna, ${ }^{1,2}$, Sami J. Barmada ${ }^{8}$, Leeanne McGurk ${ }^{3}$ and May Khanna ${ }^{1,2^{*}}$

From the ${ }^{1}$ Department of Pharmacology, College of Medicine, University of Arizona, Tucson, AZ 85724, USA; ${ }^{2}$ Center for Innovation in Brain Science, Tucson, AZ 85721, USA; ${ }^{3}$ Cell and Developmental Biology, School of Life Sciences, University of Dundee, Dow Street, Dundee DD1 5EH, UK; ${ }^{4}$ Enabling Technologies Group, Sanford Research, Sioux Falls, SD; ${ }^{5}$ Pharmacology and Toxicology, School of Pharmacy, University of Arizona, Tucson, AZ 85724, USA; ${ }^{6}$ Department of Biochemistry and Biophysics, Perelman School of Medicine, University of Pennsylvania, Philadelphia, PA 19104, USA; ${ }^{7}$ Department of Pediatrics, Sanford School of Medicine, University of South Dakota, Sioux Falls, SD; ${ }^{8}$ Department of Neurology, University of Michigan, Ann Arbor, MI 48109

\footnotetext{
* To whom correspondence should be addressed: Dr. May Khanna, Department of Pharmacology, College of Medicine, University of Arizona, 1501 North Campbell Drive, P.O. Box 245050, Tucson, AZ 85724, USA Office phone: (520) 626-2147; Fax: (520) 626-2204; Email: maykhanna@email.arizona.edu.
}

Keywords: TDP43; Hsp70; HspA5; BiP; GRP78; binding interface; ALS; chaperone 


\section{ABSTRACT}

Amyotrophic lateral sclerosis (ALS) is a fatal neurodegenerative disease with no cure or effective treatment in which TAR DNA Binding Protein of $43 \mathrm{kDa}$ (TDP-43) abnormally accumulates into misfolded protein aggregates in affected neurons. It is widely accepted that protein misfolding and aggregation promote proteotoxic stress. The molecular chaperones are the body's primary line of defense against proteotoxic stress and there has been long-standing interest in understanding the relationship between chaperones and aggregated protein in ALS. Of particular interest are the heat shock protein of $70 \mathrm{kDa}$ (Hsp70) family of chaperones; however, defining which of the 13 human Hsp70 isoforms is critical for ALS, has presented many challenges. To gain insight into the specific Hsp70 that modulates TDP-43, we investigated the relationship between TDP-43 and the Hsp70s using proximity-dependent biotin identification (BioID) and discovered several Hsp70 isoforms associated with TDP-43 in the nucleus, raising the possibility of an interaction with native TDP-43. We further found that HspA5 bound specifically to the RNA-binding domain of TDP-43 using recombinantly expressed proteins. HspA5 is increased in prefrontal cortex neurons of ALS patients. Finally, overexpression of HspA5 in Drosophila rescued TDP-43-induced toxicity, suggesting that upregulation of HspA5 may have a compensatory role in ALS pathobiology. 


\section{INTRODUCTION}

Proteostasis is the proper equilibrium between the biogenesis, folding, trafficking and degradation of proteins within the cellular milieu ${ }^{1}$. Any interference in proteostasis leads to accumulation of misfolded proteins, a central pathological hallmark of several neurodegenerative diseases including Alzheimer's disease and amyotrophic lateral sclerosis (ALS) ${ }^{2,3}$. In over $95 \%$ of ALS patients, TAR DNA-binding protein of $43 \mathrm{kDa}$ (TDP-43) is mislocalized from the nucleus to the cytoplasm where it is found misfolded and aggregated in affected neurons and glia ${ }^{1,2}$. TDP-43 pathology has been observed across several neurodegenerative disorders including frontotemporal degeneration (FTD), Alzheimer's disease, and limbic-predominant age-related TDP-43 encephalopathy (LATE $)^{4-7}$. Although the causative factors that lead to TDP-43 aggregation are still not fully understood, studies implicate proteostasis mechanisms such as impaired autophagy and the ubiquitin proteasome system (UPS) ${ }^{8,9}$ as well as compromised endolysosomal function ${ }^{10-12}$. TDP-43, a DNA/RNA-binding protein, consists of a folded N-terminal domain (NTD) linked by a flexible loop to two tandem RNA recognition motifs (RRMs) - RRM1 and RRM2 - and a predominantly unfolded C-terminal prion-like domain that harbors the majority of diseaseassociated mutations in $\mathrm{ALS}^{13}$. TDP-43 functions primarily in RNA metabolism including splicing, translation and the cytoplasmic stress granule response ${ }^{14}$. Thus, in ALS, TDP-43 aggregation leads to a loss of function effect on TDP-43-controlled pathways as well as a dysregulation of proteostasis ${ }^{15,16}$.

Central to proteostasis are the chaperones; a large family of proteins that typically bind to exposed hydrophobic sequences to assist in protein misfolding, degradation and the clearance of aggregated protein $^{17,18}$. One major chaperone subfamily are the evolutionary conserved Hsp70s, which consist of 13 gene products (HspA1A, HspA1B, HspA1L, HspA2, HspA5, HspA6, HspA7, HspA8, HspA9, Hsp12A, Hsp12B, Hsp13 and Hsp14) $)^{19,20}$. The canonical Hsp proteins share high sequence identity and have diverse cellular localizations and functions ${ }^{19}$. All canonical Hsp70 proteins have an N-terminal nucleotide binding domain (NBD) and a C-terminal substrate-binding domain (SBD) that allosterically communicate in an ATP-dependent manner to recognize and bind client proteins ${ }^{21}$. 
Typically, high levels of Hsp70 can be produced by cells in response to hyperthermia, oxidative stress, changes in $\mathrm{pH}$, chemical disruption of proteostasis ${ }^{22}$ and expression of disordered proteins $\mathrm{s}^{23-25}$. Intriguingly, in motor neurons, the primary cells affected in ALS, there appears to be an incomplete Hsp stress response, as inferred from the lack of Hsp70 upregulation in response to several stress paradigms ${ }^{26,27}$. Moreover, overexpression of chaperones, including Hsp70s, reduced TDP-43 aggregate formation ${ }^{28}$ and injection of recombinant human Hsp70 was effective in improving motor defects as well as increasing lifespan of a superoxide dismutase type 1 (SOD1) mouse model of $\mathrm{ALS}^{29}$. Collectively, these findings may partially explain why strategies to boost Hsp70 have been touted as neuroprotective in neurodegenerative diseases, particularly ALS. In support of this, Arimoclomol, a co-inducer of heat shock protein expression, has been under investigation in a clinical trial for ALS patients but recently failed in phase II/III (Clinicaltrials.gov identifier NCT03491462). Arimoclomol is known to prolong heat shock factor 1 (HSF1) binding to the heat shock element (HSE) localized in the promoter of inducible Hsp70 isoforms, and induced the expression of a certain subset of heat shock proteins in neuronal cell lines ${ }^{30}$. This might indicate that only a precise Hsp70 isoform subset is able to mitigate ALS toxicity.

It is still unclear how and which Hsp70 isoforms regulate TDP-43. Previous studies demonstrate that at least three Hsp70 isoforms immunoprecipitate with TDP-43: HspA1A, HspA5 and HspA8 ${ }^{31}$. It was later hypothesized that Hsp70s could be constitutively bound to TDP-43. Upon a heat shock event, Hsp70 could be released from its interaction with TDP-43, as misfolded proteins accumulate, which could thereby promote the formation of TDP-43 aggregates ${ }^{32}$. More recently, it was shown that in cells, several Hsp70 isoforms accumulate within mutated TDP-43 phase separated anisosomes (an anisotropic intranuclear liquid spherical shell) ${ }^{33}$. To date, potential direct binding between the Hsp70 isoforms and TDP-43 has not been investigated. Here, we interrogated the association of TDP-43 with Hsp70 isoform using BioID, a technique that leverages the activity of a promiscuous biotin ligase to biotinylate proteins based on proximity $^{34}$. We found that HspA5 and HspA8 were enriched in the nuclear, but not cytoplasmic, fraction of TDP-43. We further tested direct binding of TDP-43 with the Hsp70 isoforms HspA1A, HspA5 and 
HspA8 and found that the TDP-43 RRM domains selectively bind HspA5. We showed an upregulation of HspA5 in neurons of the prefrontal cortex of ALS patients compared to healthy controls. Finally, we discovered that upregulation of the HspA5 homologue (or Hsc70.3 in Drosophila melanogaster) protects against TDP-43-induced degeneration in Drosophila while the ATP binding-deficient mutant Hsc70.3 ${ }^{\text {K97S }}$ variant $^{35}$, had no effect. Our data underscore Hsp70 isoform preference by TDP-43 and thus position induction of HspA5 binding to TDP-43 as a novel therapeutic strategy for mitigating TDP-43 toxicity.

\section{Results}

\section{BioID identifies Hsp70 networks binding to TDP-43 in the nucleus.}

To characterize nuclear versus cytoplasmic localization as well as possible Hsp70 isoform specificity of TDP-43, we performed proximity-dependent biotin labeling (BioID) of TDP-43 in the nucleus or the cytoplasm. BioID2 was fused to the N-terminal domain of TDP-43, and either a 3x tandem nuclear localization signal (3xNLS) or a nuclear export signal (NES) was added to localize TDP-43 to the nucleus or cytoplasm, respectively. BioID2-3xNLS-TDP43, BioID2-NES-TDP43 or the BioID2 control were stably expressed in human neuroblastoma SH-SY5Y cells and their localization was verified using immunofluorescence (Fig. 1A). It is worth noting that while BioID2-NES-TDP43 mostly localized to the cytoplasm, some marginal nuclear localization was observed and is likely due to the intrinsic NLS of TDP43. Cells expressing each TDP43 variant or BioID2-only as a control were lysed for BioID pulldown in triplicate, and affinity capture of biotinylated proteins was confirmed via western blot (Fig. S1). Biotinylated proteins identified via mass spectrometry (MS) were ranked by label-free quantification (LFQ) intensity, enrichment compared to BioID2-only control, and the number of replicates $(\mathrm{N})$ of each protein was identified. Following a criterion of 3 -fold enrichment over control and $\mathrm{N} \geq 2$ threshold, 144 nuclear and 28 cytoplasmic interaction candidates for TDP-43 were identified (Fig. 1B, C, Table S1). "Highest confidence associations" were proteins found only in the BioID2-3xNLS-TDP43 or BioID2-NES-TDP43 samples, and not at all in the control BioID samples, and ranked by LFQ intensity (Fig. 1B). "Good 
confidence associations" were proteins enriched at least 3-fold over control, ranked by experimental: control intensity ratio (Fig. 1C).

Surprisingly, HspA5 and HspA8 were found as highest confidence and good confidence associations respectively in the nuclear TDP-43 sample (BioID2-3xNLS-TDP-43) (Fig. 1B, C). No Hsp70 isoform was identified in the cytoplasmic TDP-43 sample (BioID2-NES-TDP43), hinting toward an absence of such an interaction with TDP-43 in the cytoplasm without stress (Table S1). HspA8 is well described for its implication in nuclear import of client proteins as it shuttles between the cytoplasm and nucleus ${ }^{36}$. Although HspA5 is mostly known for its ER localization, several studies have shown the presence of HspA5 in the nucleus ${ }^{33,37,38}$, including in SH-SY5Y cells ${ }^{39}$. Thus, our data suggest that in the SH-SY5Y cells and in the absence of stress, HspA5 and HspA8 selectively associate with nuclear but not cytoplasmic, TDP-43.
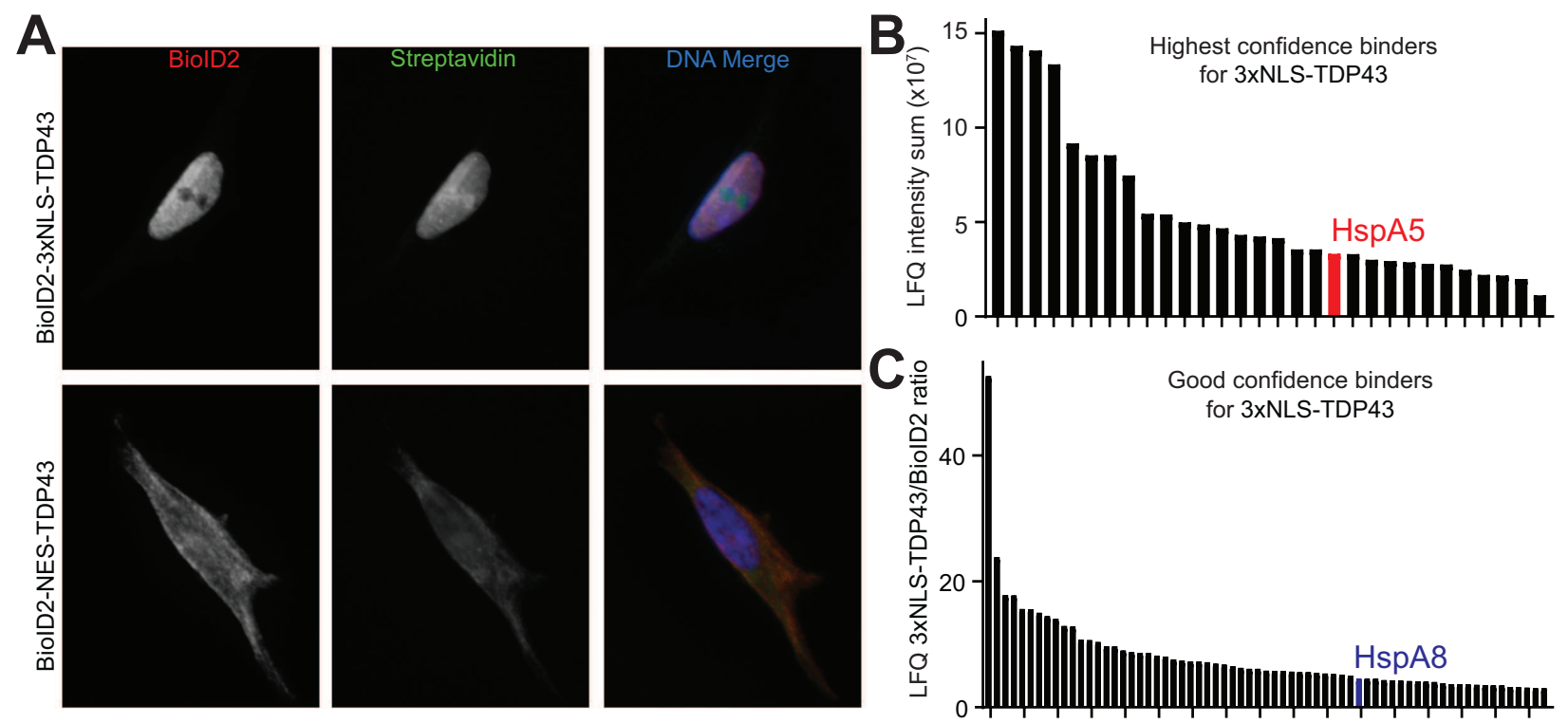

Figure 1: BioID of TDP-43 in SH-SY5Y cells in the nucleus and cytoplasm. A. Epifluorescence images for validating fusion-protein (red) expression and promiscuous biotinylation (green) localization following the addition of biotin. The 144 nuclear interactions of the 3xNLS-TDP-43 sample were separated into "Highest confidence associations", proteins found only in the BioID2-3xNLS-TDP43, and not at all in the 
control BioID samples, and ranked by label-free quantification (LFQ) intensity (B); $\boldsymbol{C}$. "Good confidence associations" were proteins enriched at least 3-fold over control, ranked by experimental:control intensity ratio.

\section{The RRM domains of TDP-43 selectively bind to the Hsp70 isoform HspA5.}

The BioID data hinted towards TDP-43 binding selectively to specific Hsp70 isoforms, as demonstrated by the fact that only HspA8 and HspA5 were found to be significantly enriched. Another previous study showed via immunoprecipitation that Hsp70 interacts with TDP-43 primarily through its $\mathrm{RRMs}^{33}$, but the exact Hsp70/TDP-43 interface was never investigated. We thus set out to characterize the binding of TDP-43 to different Hsp70 isoforms. To this end, we selected HspA5 and HspA8 (identified from BioID) and HspA1 A, an Hsp70 isoform implicated in TDP-43 binding ${ }^{31}$.

We thus first predicted where Hsp70 could bind to TDP-43 using LIMBO, a position specific algorithm for identifying Hsp70 binding sites in proteins ${ }^{40}$. LIMBO is based on a position-specific scoring matrix (PSSM) trained from in vitro peptide binding data and structural modelling and predicts the binding of bacterial Hsp70 homolog DnaK, which shares $~ 50 \%$ identity with human Hsp70 isoforms. For Hsp70 prediction, TDP-43 was divided into three fragments: aa 1-120 (N-terminal domain and flexible linker of TDP-43 (NTD)), aa 101-269 (the two RNA recognition motifs (RRM)) and aa 270-414 (C-terminal prionlike domain, which is mostly unstructured, aggregation prone, and the site for most ALS mutations) (Fig. S2A). While predicted binding sites were noted in the NTD and RRM domains, the algorithm did not predict any Hsp70 binding sites in the prion-like domain (Fig S2B, C). Thus, our computational predictions suggest that the HSP70 does not bind to the unstructured C-terminal domain but to the N-terminal domain and to the RRMs.

Using Microscale Thermophoresis (MST), we measured the binding of the substrate binding domain (SBD) of HspA1A, HspA5 and HspA8 to TDP-43 $1-102$, a construct corresponding to TDP-43-NTD. The SBD of these Hsp70 isoforms is approximately 200 amino acids long and is composed of a two layered 
twisted $\beta$-sheet and a $\mathrm{C}$-terminal $\alpha$-helical subdomain. The SBD and its binding to the client peptide is allosterically modulated by the ATP binding site. However, binding of ATP to the TDP-43 RRM domains has also been shown to enhance the stability of TDP- $-43^{41}$, thus we reasoned that this may inhibit Hsp70 isoform binding, and we opted for an Hsp70 construct that lacked the N-terminal nucleotide binding site but retained the ability to recognize client peptides.

All three Hsp70 isoforms bound TDP-43 $3_{1-102}$ with a similar affinity calculated to be in the high nanomolar to low micromolar range (Fig. 2A, B). There was a small but significant difference in the binding affinity between the between binding of HspA1A and HspA5 to TDP-43 ${ }_{1-102}(p=0.0242$, Fig. 2B). By contrast, in MST experiments with the RRM domain (TDP-43 ${ }_{102-269}$ ), we found that HspA8 did not bind at all, and that HspA5 $(549 \pm 68 \mathrm{nM})$ bound with $\sim 6$ fold greater affinity than $\operatorname{HspA} 1 \mathrm{~A}(3.2 \pm 2.3 \mu \mathrm{M})($ Fig. 2C, D). TDP-43 ${ }_{102-269}$ binding to HspA5 exhibited a significantly lower Kd than HspA1A (p = 0.0002; Fig. 2D), indicating that TDP-43102-269 was selective for HspA5 over the other isoforms tested. Overall, our data indicate that while the unstructured NTD of TDP-43 binds to HspA1A, HspA5 and HspA8 with almost equal affinity; the conformationally stable RRM domains of TDP-43 have a greater propensity to bind HspA5.
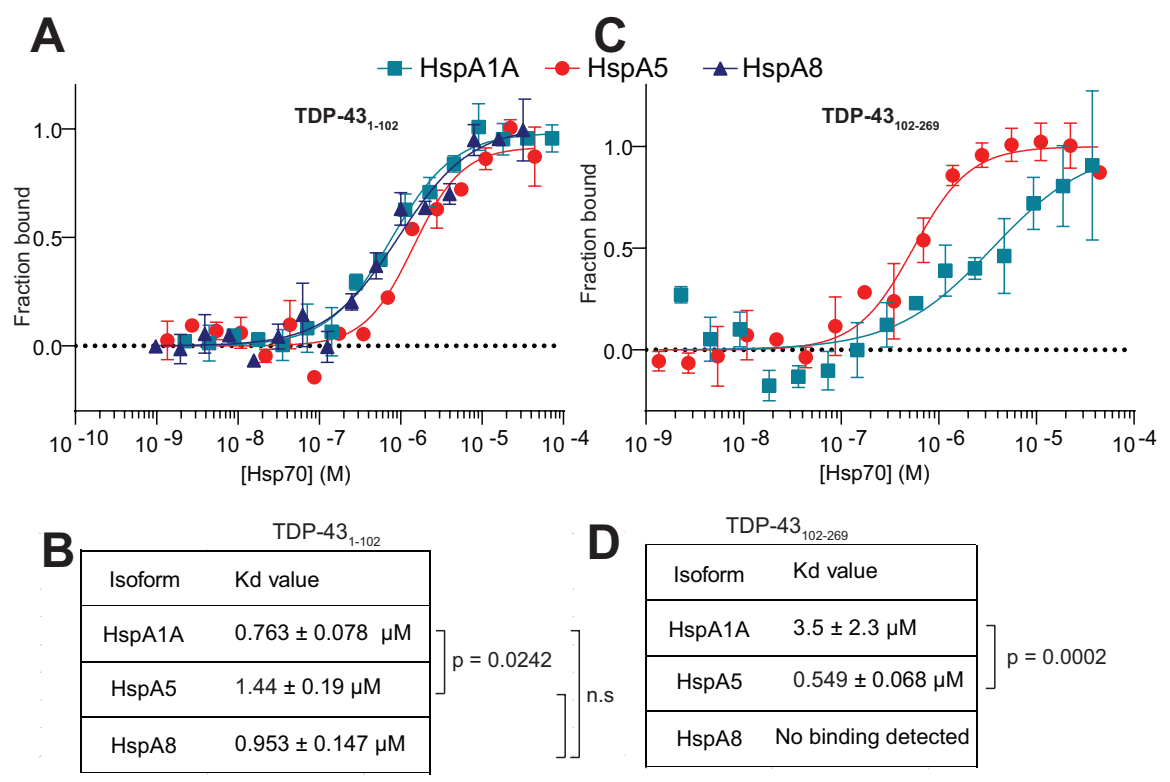
Figure 2: Selective binding of native TDP-43 constructs to Hsp70 isoforms. Microscale thermophoresis was used to measure the binding of ranging concentrations of Hsp70 SBDs to $50 \mathrm{nM}$ of labelled TDP-43 ${ }_{1-}$ $102(\boldsymbol{A})$ or TDP-43 $3_{102-269}(\boldsymbol{C})$. HspA1A and HspA5 were able to bind TDP-43 ${ }_{102-269}$. No signal was detected for HspA8 binding to TDP-43 102-269. B. Table of affinity constants extracted from the MST values for TDP43 1-102. Statistical difference was assessed between HspA1A, HspA5 and HspA8 binding (Brown-Forsythe and Welch ANOVA test). D. Table of affinity constants extracted from the MST values for TDP-43102-269. Statistical difference was assessed between HspA1A and HspA5 binding (Welch's test). Data is presented as Mean $\pm S D(n=3)$.

\section{HspA5 binds TDP-43 RRM2 at the interface with RNA}

Spurred by the selective binding of the RRM region of TDP-43 (TDP-43 ${ }_{109-260}$ ) to HspA5 we set out to experimentally map, in greater resolution, potential HspA5 binding sites within TDP-43. To do this we synthesized a peptide-binding array of 15-mer peptides with an overlap of 5 amino acids that spanned the RRM region of TDP-43. The peptide binding array was incubated with HspA5-SBD protein and peptide binding was detected using an antibody directed against HspA5 (Fig. 3A). HspA5 bound to several TDP43 peptides in RRM1 (noted in red in Fig. 3A) and in RRM2 (highest binding peptide shown in orange in Fig. 3A). Some C-terminal TDP-43 peptides also bound to HspA5, but this could be due to the fact that these C-terminal peptides (e.g., peptide 70) have several glutamine (Q) and asparagine (N) amino acids, typical of prion-like domains. There was good concordance between our computationally predicted sites (Fig. S2B, C) and peptides in the RRM1 and RRM2 domains of TDP-43 that bound HspA5.

We next mapped these potential HspA5-binding regions on TDP-43 ${ }_{102-269}$ in the context of the 3dimensional and folded structure of TDP-43. We calculated the surface accessibility of the TDP-43 peptides bound by Hsp70 and mapped the peptide sequence on to the known TDP-43 structures of the RRM domains

complexed to (UG) 6 RNA (PDB code: 4bs2 ${ }^{42}$ ) (Fig. 3). Notably, all of the TDP-43 peptides bound by HspA5 in the NTD and RRM domains have partial surface accessibility (Fig. 3B). Moreover, they have 
relatively low dynamics in the NMR structures and include secondary structural elements (helix for the accessible peptide in RRM1, strand for the accessible peptide in RRM2) (Fig. 3C). Given that HspA5 binds TDP-43 $3_{102-269}$, these data suggest that (i) HspA5 might recognize only a portion of the peptide, sufficient for initiating a binding, and (ii) there might be structural elements at play in the HspA5/TDP-43 interaction.

Interestingly, one TDP-43 client peptide (shown as red in Fig. 3) overlaps with the ribonucleic protein motif-2 (LIVLGL in RRM1). We tested if RNA had an effect on binding using increasing concentrations of $\mathrm{UG}_{6}$ RNA, the canonical binding sequence of TDP- $43^{43}$, and we detected a decreased affinity of the HspA5/TDP-43 $3_{102-269}$ interaction from $0.9 \pm 0.3 \mu \mathrm{M}$ to $28.3 \pm 23.7 \mu \mathrm{M}$ (Fig. 3E). RNA binding is thought to maintain TDP-43 in a soluble state, and has been proposed to prevent passive exit from the nucleus ${ }^{44,45}$. A second client peptide (shown as red in Fig. 3) is adjacent to amyloidogenic sequences in RRM2 (246-EDLIIKGISV-255; shown in orange in Fig. 3) ${ }^{46}$. Since exposed E246/D247 residues are markers of misfolded TDP- $43^{47}$, exposing client peptides following loss of nucleic acid binding and/or exposure of amyloidogenic regions, might trigger HspA5 association to prevent aggregation of TDP43. 

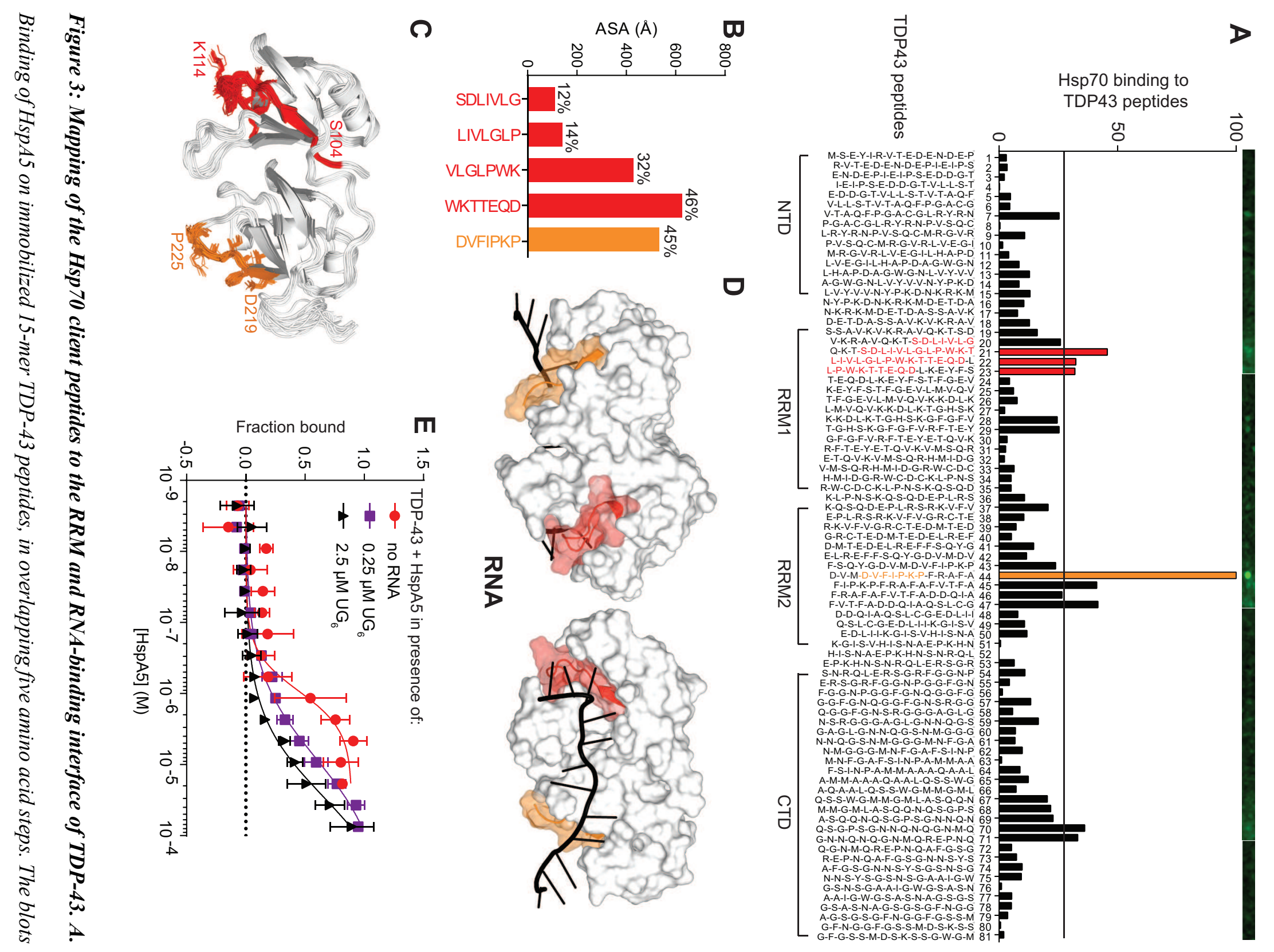
were scanned, and spot intensities were quantified and represented as a normalized signal. Highly scored LIMBO predicted client peptides of TDP-43 are shown in red and orange, respectively. B. Accessibility of the client peptides from the peptide array at the TDP-43 surface (ASA) were calculated using Areaimol as implemented in the CCP4 suite ${ }^{35}$ on the free form structure of the tethered RRM domains (PDB ID $4 b s 2^{22}$ ).

To note, there is no significant difference in accessibility values when RNA is present. The percentage of accessibility represents the ASA of the motif compared to the total surface of TDP-43. C-D. Mapping of the client peptides on TDP-43 or NMR structures (cartoon representation) (C) or surface (D) of the RRM domain (PDB code: $4 b s 2^{22}$ ). The predicted client peptides were color coded as described in A. $\boldsymbol{E}$. Microscale thermophoresis of NTA-labelled TDP-43102-269 interaction with HspA5 in the absence or in the presence of increasing concentrations of $U G_{6} R N A$. The presence of $R N A$ shifted the Kd of the TDP-43 ${ }_{102-}$ ${ }_{26} /$ HspA5 interaction from $0.89 \pm 0.25 \mu M$ (red curve) to $28.3 \pm 23.7 \mu M$ (black curve). Data is presented as Mean $\pm S D(n=3)$.

\section{Hsp70A5 is increased in the cytoplasm in human ALS tissue}

Considering the interaction between TDP-43 and HspA5, as well as the mislocalization of TDP-43 in ALS, we next asked whether the distribution of HspA5 is affected in ALS patients. To do this we immunolabelled paraffin-embedded sections of the layer $\mathrm{V}$ of frontal cortex of 2 control patients and 2 sporadic ALS patients for the HspA5 protein. In control patients, HspA5 exhibits a granular, cytoplasmic pattern within neurons (Fig. 4A). Although the general distribution of HspA5 (granular and cytoplasmic) remains unchanged in the neurons of the frontal cortex of the 2 ALS patients, the immunoreactivity of HspA5 was increased in intensity (Fig. 4B). Our data suggest, that in the neurons of the frontal cortex of ALS patients, the HspA5 protein is upregulated in the cytoplasm. 

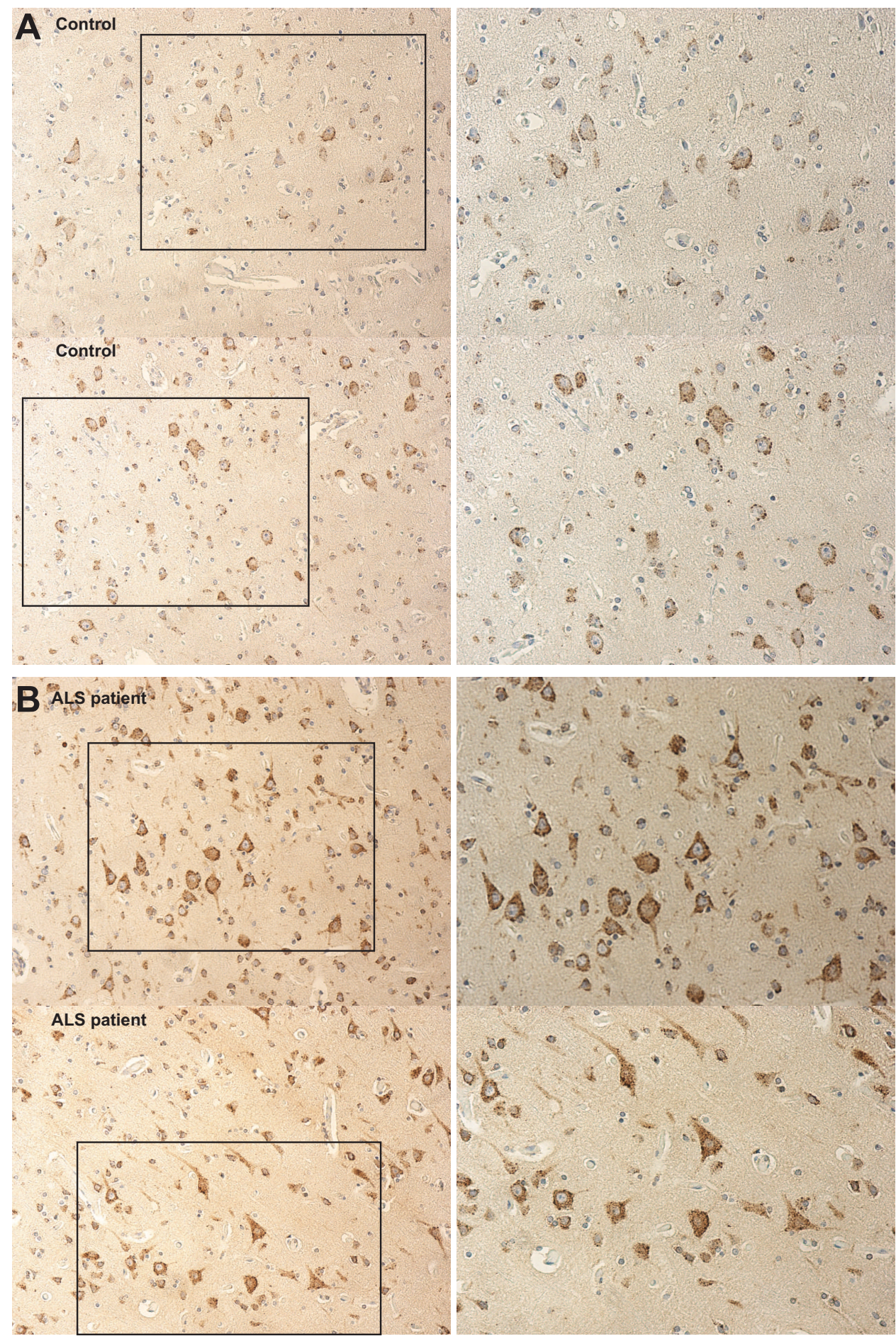

Figure 4: HspA5 is overexpressed in the cytoplasm of neurons from prefrontal cortex of ALS patients.

Deep (layer V) neurons of the frontal cortex were immunostained for HspA5, and counterstained with hematoxylin. A. ALS patients; B. control patients. Right panels are zoomed in version of left panels. 


\section{Upregulation of the HspA5 Drosophila homolog mitigates TDP-43 disease-associated toxicity.}

Since we found a direct and specific interaction of HspA5 with native TDP-43 as well as an overexpression of this Hsp70 isoform in ALS neurons, we set out to determine if HspA5 could modulate TDP-43 toxicity using a Drosophila model of TDP-43 toxicity ${ }^{48,49}$. Expression of human TDP-43 in the Drosophila eye disrupts the external surface (compare $0 \%$ to $61.7 \% \pm 6.9 \%$, normal vs TDP-43 respectively (Fig. 5A-B)), reduces retinal width (compare $73.3 \pm 3.8 \mu \mathrm{m}$ to $30.3 \pm 7.7 \mu \mathrm{m}$, normal vs TDP43 respectively (Fig. 5A-B)) and causes retinal vacuolization (compare $78.1 \mu^{2}$ to $3469 \mu \mathrm{m}^{2}$, normal vs TDP-43 respectively (Fig. 5A-C)) - all of which are readouts of TDP-43-associated toxicity.

The Drosophila HspA5 homologue is Hsc70.3 (Fig. S3A-B). In the absence of TDP-43, upregulation of the normal form of Hsc70.3 $\left(\mathrm{Hsc} 70.3^{\mathrm{WT}}\right)$ had no effect on the Drosophila eye, however, in contrast, downregulation of Hsc70.3 altered the structure of the external eye indicating that loss of Hsc70.3 is detrimental to the Drosophila eye (Fig. S3C). We thus focused on the effect of upregulating Hsc70.3 on TDP-43 toxicity. Co-expression of TDP-43 with Hsc70.3 ${ }^{\text {WT }}$ in the Drosophila eye significantly reduced the external and internal degeneration induced by TDP-43 (Fig. 5A-C). To address whether the suppression of TDP-43 toxicity by Hsp70.3 upregulation required ATPase activity we expressed Hsc70.3 variants with defective ATPase activity (Hsc70.3 $3^{\mathrm{D} 31 \mathrm{~S}}$ and $\left.\mathrm{Hsc} 70.3^{\mathrm{K} 97 \mathrm{~S}}\right)^{35}$ in the Drosophila eye and selected the variant that conferred no toxicity $\left(\mathrm{Hsc} 70.3^{\mathrm{K} 97 \mathrm{~S}}\right)$. Co-expression of Hsc $70.3^{\mathrm{K} 97 \mathrm{~S}}$ with TDP-43 had no effect on TDP43-induced degeneration of the external eye and retinal width but suppressed TDP-43-induced vacuolization (Fig. 5B-D, Fig. S3C), indicating that Hsc70.3 requires ATPase activity to fully suppress TDP-43 toxicity. Importantly, upregulation of $\mathrm{Hsc} 70.3^{\mathrm{WT}}$ or Hsc70.3 ${ }^{\mathrm{K} 97 \mathrm{~S}}$ had no effect on the total protein levels of TDP-43 or a control protein ( $\beta$-galactosidase) (Fig. 5E-F, Fig. S4), indicating that the suppression of TDP-43 by Hsc70.3 is not simply because of reduced TDP-43 protein levels. Collectively, our data indicate that upregulation of Hsc70.3 is beneficial in preventing TDP-43-associated toxicity in Drosophila. 
A

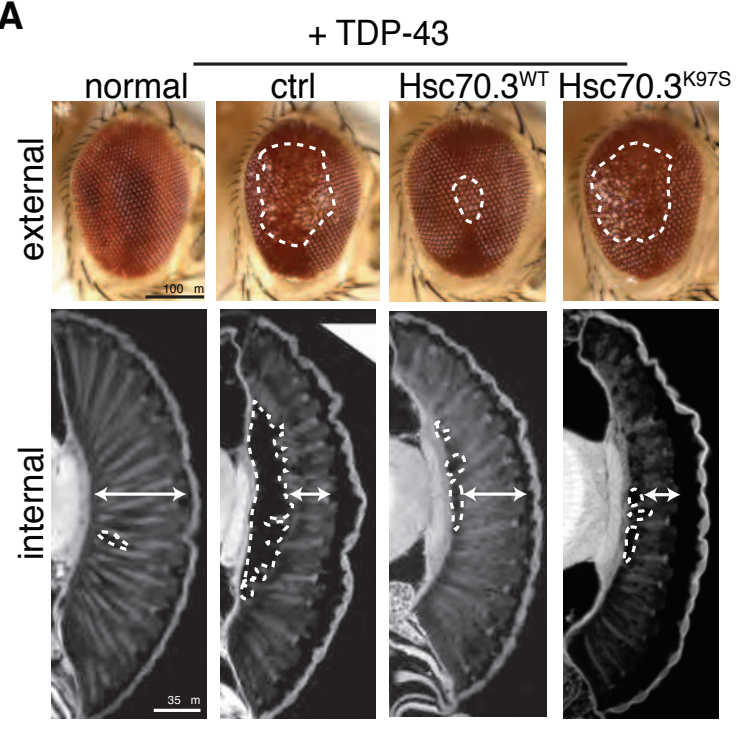

B

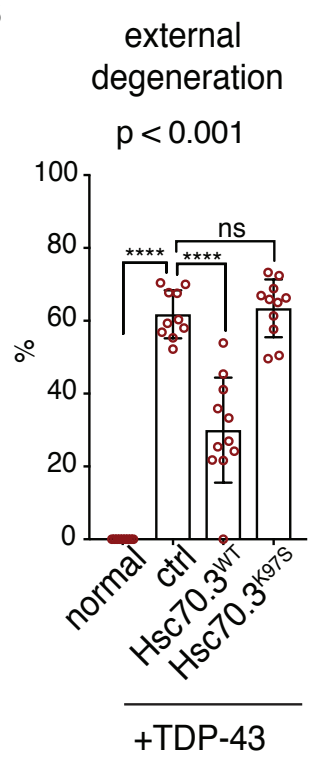

C

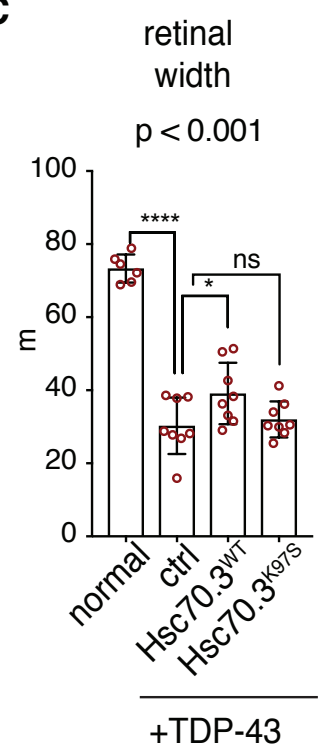

D

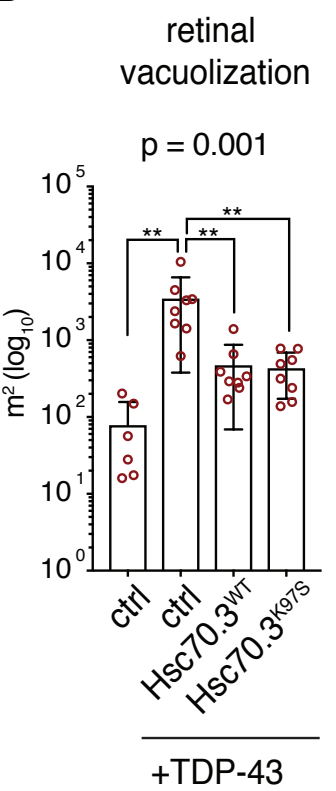

E

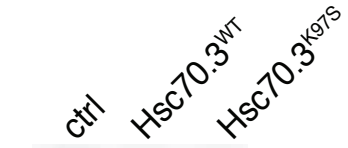
50 -
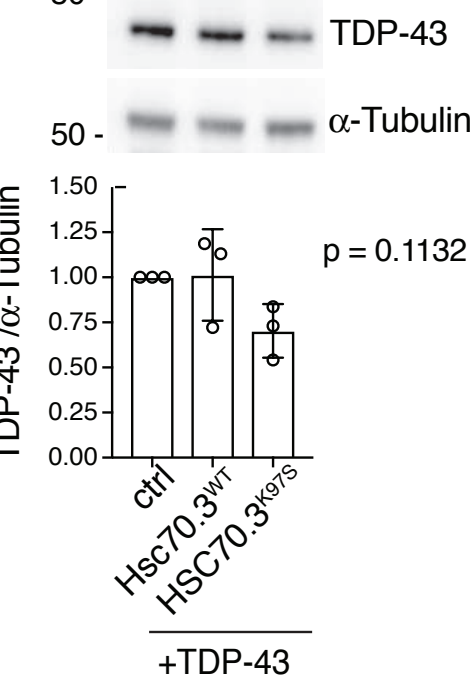
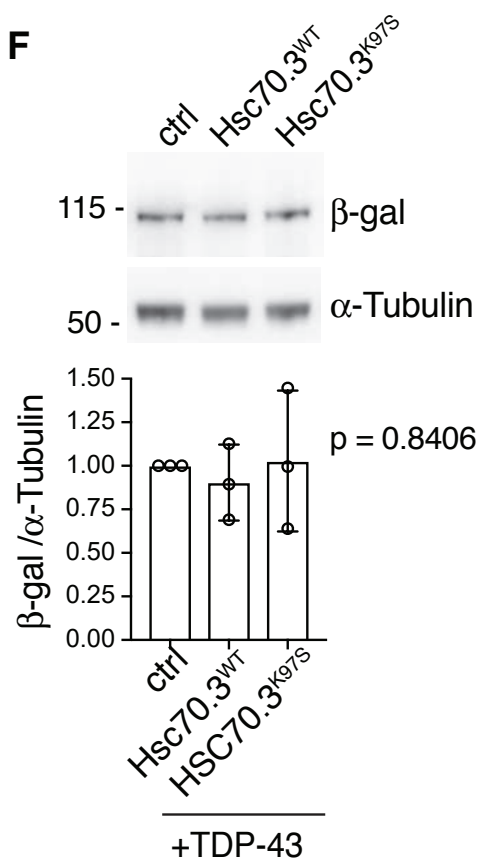

Figure 5: Hsc70.3 genetically interacts with TDP-43 in the Drosophila eye. A. Compared to the normal control, expression of human TDP-43 (ctrl) in the Drosophila eye leads to disruption of the external eye (white hatched line, top panel) and degeneration of the internal retina (white double headed arrow, and white hatched line lower panel). B. Expression of Hsc70.3 suppresses TDP-43-induced disruption of the external eye. Mean $( \pm \mathrm{SD})$, one way ANOVA and a Fisher's LSD test. ****: $\mathrm{P}<0.0001$ and ns: not significant. C. Expression of Hsc70.3 ${ }^{\mathrm{WT}}$ suppresses TDP-43-induced reduction of retinal width (see double 
headed arrow, lower panel in $(\mathrm{A}))$. Mean $( \pm \mathrm{SD})$, one way ANOVA and a Fisher's LSD test. ****: $\mathrm{P}<0.0001, *: \mathrm{P}<0.05$ and ns: not significant. D. Expression of $\mathrm{Hsc} 70.3^{\mathrm{WT}}$, and $\mathrm{Hsc} 70.3^{\mathrm{K} 97 \mathrm{~s}}$ reduces TDP43 -induced vacuolization of the internal eye (white hatched line, lower panel in $(A))$. Mean $( \pm S D)$, one way ANOVA and a Fisher's LSD test. ****: $\mathrm{P}<0.0001, *: \mathrm{P}<0.05$ and ns: not significant. E. Upregulation of Hsc $70.3^{\text {WT }}$ or Hsc70. $3^{\text {K97S }}$ had no effect of the total protein levels of TDP-43. Protein isolated from $\sim 5-10$ male heads immunoblotted for TDP-43 and Tubulin. Protein levels were quantified from 3 independent biological repeats. Graph represents the mean $\pm \mathrm{SD}$, one-way ANOVA and Tukey's test, ns: not significant. F. Upregulation of Hsc70.3 $3^{\mathrm{WT}}$ or Hsc70.3 ${ }^{\mathrm{K} 97 \mathrm{~S}}$ had no effect of the total protein levels of $\beta$-galactosidase. Protein isolated from $\sim 5-10$ male heads immunoblotted for $\beta$-galactosidase and Tubulin. Protein levels were quantified from 3 independent biological repeats. Graph represents the mean $\pm \mathrm{SD}$, one-way ANOVA and

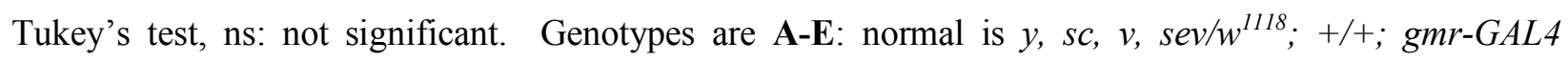
$(Y H 3) / s i . m C h e r r y y^{35783}, \operatorname{ctrl}$ is $y, s c, v, s e v / w^{1118} ; U A S-T D P-43 /+; \operatorname{gmr}-G A L 4$ (YH3)/ si.mCherry ${ }^{35783}$, Hsc70.3 ${ }^{\mathrm{WT}}$ is $w$-; UAS-TDP-43/UAS-Hsc70-3 ${ }^{W T}$; gmr-GAL4 $(Y H 3) /+$ and Hsc70.3 ${ }^{\mathrm{K} 97 \mathrm{~S}}$ is $w$-; UAS-TDP-

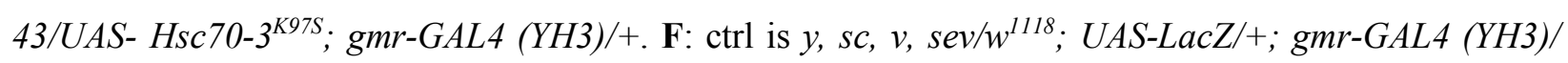
si.mCherry ${ }^{35783}, \mathrm{Hsc} 70.3^{\mathrm{WT}}$ is $w$-; UAS-LacZ/UAS-Hsc70-3 ${ }^{W T}$; gmr-GAL4 (YH3)/+ and Hsc70.3 ${ }^{\mathrm{K} 97 \mathrm{~s}}$ is $w$-; UAS-LacZ/UAS-Hsc70-3 ${ }^{K 97 S}$; gmr-GAL4 (YH3). 


\section{Discussion}

Targeting the molecular chaperone pathway is a potential therapeutic strategy in neurodegenerative disorders such as ALS. Arimoclomol, a compound that increases Hsp70 proteins as well as other Hsp chaperones $^{50}$, recently failed phase II/III clinical trials for the treatment of ALS (Clinicaltrials.gov identifier NCT03491462). A greater understanding of which HSP family members control TDP-43 will be crucial for insights into the mechanisms that propagate disease as well as in developing more nuanced therapeutic strategies. Here we show that the Hsp70 isoform HspA5 specifically binds to the RNA-binding domain of TDP-43, that there is an apparent increased expression of cytoplasmic HspA5 in the motor cortex of ALS patients and that upregulation of the HspA5 homologue mitigates TDP-43-induced toxicity in Drosophila, identifying HspA5 as a potential target in TDP-43-associated disease.

Although a plethora of proteins and protein families have been reported to interact with and control TDP-43, they have generally been identified using indirect measures such as genetic interaction screens $^{48,49,51-56}$ and affinity pull-down methods ${ }^{31,55,57,58}$. Here, using BioID, a unique technique that leverages promiscuous nature of biotin ligase to biotinylate proteins based on proximity ${ }^{34}$, only HspA5 and HspA8 were found to bind TDP-43 in the nucleus and in the absence of exogenous stress. HspA5 and HspA8 are constitutively expressed, contrary to HspA1A expression, for example, that is only induced by different stressors. Moreover, even though both HspA5 and HspA8 are commonly known to reside within the endoplasmic reticulum and the cytoplasm respectively, HspA5 is actively translocated to other cellular locations, including mitochondria and the nucleus ${ }^{39,59,60}$, and cytoplasmic HspA8 shuttles between cytoplasm and nucleus, which enables it to import client proteins in the nucleus ${ }^{61}$. Moreover, several Hsp70 isoforms, including HspA5 and HspA8, were found accumulated within mutated TDP-43 phase separated anisosomes (anisotropic intranuclear liquid spherical shells) ${ }^{33}$. Overall, this supports the possibility of a TDP-43/Hsp70 isoform interaction in the nucleus, and while an interaction with Hsp70 chaperones in the cytoplasm was not observed here it is possible that such an interaction may happen upon activation of the stress response. 
Using in vitro binding approaches, we further established that HspA1A, HspA5 and HspA8 bind directly to TDP-43. Our data indicate that while the Hsp70 isoforms HspA1A, HspA5 and HspA8 bind to the partially or fully unfolded N-terminal domain of TDP-43 with equal affinities, binding to the conformationally stable RRM domains of TDP-43 is highly selective for HspA5. Using a peptide-binding array we identified HspA5 binding sites in each RRM of TDP-43. The HspA5 binding regions in the RRMs are only partially exposed, which is surprising since chaperones typically recognize hydrophobic stretches of amino acids in unfolded proteins ${ }^{17}$. It is thus possible that the recognition of the RRM domains of TDP43 by HspA5 needs structural elements in addition to the predicted Hsp70 binding sites and perhaps may be involved in an alternative function to chaperone activity. In the absence of stress, HspA5 maintains the three transmembrane UPR sensors (PERK, IRE1 and ATF6) in an inactive state through direct binding to the respective proteins ${ }^{62}$. Upon ER stress, accumulated misfolded proteins titrate HspA5 away from PERK/IRE1/ATF6, leading to their activation and subsequent stimulation of the UPR ${ }^{63}$. Here, we show that HspA5 binding to TDP-43 is inhibited by RNA. The importance of RNA binding to TDP-43 in maintaining TDP-43 solubility has been previously reported ${ }^{64,65}$, and our data suggest that HspA5 may recognize the non-RNA bound version of TDP-43 to ensure proper folding and/or prevent misfolding or to trap TDP-43, similarly to HspA5 binding to UPR sensors.

We have further demonstrated that upregulation of the HspA5 homologue in Drosophila protects against disease-associated toxicity of TDP-43. HSPA5 has also been implicated in regulating the toxicity and aggregation of the ALS-causing protein superoxide dismutase (SOD1). For example, knock-in mice expressing HspA5 that lacks the ER retention signal, KDEL, display age-related motor problems, loss of motoneurons and aggregation of wild-type SOD1 ${ }^{66}$. Moreover, the neuronal pathology caused by expression of mutant SOD1 (SOD1-G93A) was exacerbated in mice deficient in the HspA5 co-factor SIL1, while SIL1 overexpression induced significant neuroprotection related to improved ER proteostasis and reduced SOD1 aggregation ${ }^{67}$. It is worth noting that previous work showed that in Drosophila down regulation of tankyrase 1 and tankyrase 2 (Tnks-1/2), which physically interact with TDP-43, reduces TDP- 
43 toxicity while their upregulation enhances TDP-43 toxicity ${ }^{68}$ (also needs ref 60 (PMID:30100264) for the upregulation data). This further suggests that not all binding partners of TDP-43, when upregulated, ameliorate TDP-43 toxicity.

HspA5 mainly localizes to the endoplasmic reticulum (ER) where it controls protein folding during ER-associated stress ${ }^{69}$ but cellular stimuli such as ER stress and ER-associated degradation can lead to the localization of HspA5 to the mitochondria and the $\operatorname{cytosol}^{59,70}$. While there is little to no evidence that TDP43 functions in the ER, it was suggested that newly synthesized TDP-43 in the rough ER is transported to the nucleus ${ }^{71}$. The authors further showed an accumulation of TDP-43 in the rER in anterior horn motor neurons in ALS spinal cord tissue. ER-associated stress has been implicated in ALS pathogenesis, with HspA5, among other proteins, being upregulated ${ }^{72-74}$. It is thus also possible that HspA5 interacts with and helps to properly fold TDP-43 in the cytosol and mitochondria during ER stress, and thus HSPA5 becomes depleted in the ER leading to accumulation of newly synthesized TDP-43 in the rough ER.

Finally, it is worth noting that Arimoclomol is a co-activator that prolongs the binding of activated HSF1 to heat shock elements in the promoter region of many chaperones including Hsp70 family members. Notably, HspA5 expression is not under the control of $\mathrm{Hsfl}^{20,75-77}$. Moreover, Arimoclomol, was shown to induce the expression of only HspA6 and HspA1A in human SH-SY5Y cells ${ }^{30}$. The failure of Arimoclomol in phase II/III of clinical trial for ALS patients might be partly explained by a lack of specific Hsp70 isoform targeting.

Overall, the observations in this study suggest that upregulation of HspA5 in ALS may have a compensatory role, prolonging the survival of neurons by preventing TDP-43 misfolding and subsequent toxicity. Elucidating the stimuli and the underlying cellular mechanisms that control HspA5 binding to TDP-43 will provide the platform for investigating HspA5 as a potential therapeutic target TDP-43associated disease.

\section{Materials}


All reagents were purchased from Sigma (St. Louis, MO, USA) and Fisher Scientific (Hampton, NH) unless otherwise indicated. TDP-43 $3_{102-269}$ and TDP-43 $3_{1-102}$ were obtained as previously described ${ }^{31,32}$. The TDP-43 expression strain was described previously ${ }^{37,38}$. The Hsc70.3-WT or Hsc70.3 ${ }^{\mathrm{K} 97 \mathrm{~S}}$ Drosophila strains were obtained from the Bloomington Drosophila stock center, Indiana, USA. All fly experiments were carried out at $25^{\circ} \mathrm{C}$ in standard cornmeal molasses agar.

\section{Plasmids}

All BioID plasmids were made using In-Fusion Recombination. mycBioID pBabe (Addgene \#80901) was used as the control plasmid. TDP-43 was amplified via PCR from a pDuet TDP43 WT (purchased from Addgene, Plasmid \#27462) with an AgeI restriction enzyme (RE) site built into the 5' primer upstream of TDP-43. Amplified PCR product was inserted into mycBioID pBabe (Addgene \#80901), using XhoI and SalI RE sites. The SV40 nuclear localization signal (NLS - PKKKRKV) was inserted in tandem (3x) into the newly made BioID2-TDP43 pBabe using XhoI and AgeI. Similarly, the classic protein kinase inhibitor nuclear export signal (NES- NELALKLAGLDI) was inserted into BioID2-TDP43 pBabe using XhoI and AgeI.

\section{Methods}

Hsp70 isoforms purification. BL21-Codon plus bacteria (Agilent) were transformed with pSpeedET vectors containing Hsp70 isoform. Cells were grown to and $\mathrm{OD}_{600}$ of 0.6 at $37{ }^{\circ} \mathrm{C}$ before being shifted to $16{ }^{\circ} \mathrm{C}$. Expression was induced once the $\mathrm{OD}_{600}$ reached $0.8-1.0$ with $0.5 \mathrm{M}$ IPTG overnight. Cells were then harvested, lysed, and protein was purified using cobalt IMAC resin (Gold bio). The His 6 tag was cleaved using TEV protease overnight in dialysis into buffer A (50 mM HEPES pH 7.4, $100 \mathrm{mM} \mathrm{KCl,} 10 \mathrm{mM}$ $\mathrm{Mg}(\mathrm{OAc})_{2}$, and $1 \mathrm{mM}$ DTT $)$. After complete cleavage, the DTT was dialyzed out for 4 hours, and the TEV protease was recaptured with cobalt resin. Protein was then concentrated, flash frozen on liquid nitrogen and stored at $-80^{\circ} \mathrm{C}$. 
Microscale thermophoresis. Purified TDP43 ${ }_{102-269}$-His was labelled using the Monolith Protein Labeling Kit RED-NTA (Nanotemper, Germany) according to the manufacturer's instructions. Microscale thermophoresis experiments were performed similarly to 39 . Briefly, $50 \mathrm{nM}$ of labeled protein was mixed with ranging concentration of Hsp70 isoforms in MST buffer. The thermographs were recorded using MST premium capillaries at $40 \%$ LED and medium MST power. Data analysis was performed with the MO Affinity Analysis software (Nanotemper).

Synthesis and Blotting of SPOT Membranes. Peptides of TDP-43 (15 amino acids in length) were spotted on nitrocellulose on glass slides. Peptides were synthesized using standard 9-fluorenylmethoxycarbonyl (Fmoc) chemistry, in $30 \times 20$ spot arrays using a Multipeptide synthesizer adapted for SPOT synthesis (Intavis AG, Cologne, Germany). Membranes were blocked for at least $1 \mathrm{~h}$ in Tris-buffered saline containing $0.5 \%$ Tween 20 (TBST) with 5\% semi-skimmed milk powder before an overnight incubation with $2.5 \mu \mathrm{M}$ of $\mathrm{HspA} 5$ and $1 \mathrm{mM}$ ADP at $4{ }^{\circ} \mathrm{C}$ with gentle shaking. Following a series of washes in TBST, the blot was probed for an hour with an HspA5 antibody at $4{ }^{\circ} \mathrm{C}$. The following day, blots were washed three times for 10 min each time in TBST, incubated in secondary antibody $(\operatorname{IgG}(\mathrm{H}+\mathrm{L})$ Cross Adsorbed Secondary Antibody, DyLight 800 (ThermoFisher, Product \# SA5-10176) at dilutions 1:5,000) for 45 min at room temperature, and washed in TBST three more times for 10 min each time before visualizing SPOTs by exposing the membranes.

External eye microscopy, paraffin sectioning and quantification. For external eye imaging, female flies were imaged with a Leica Z16 Apo A microscope, DFC420 camera and 2.0x planapochromatic objective. For paraffin sections, fly heads were fixed, processed and quantified as previously described ${ }^{78} .8 \mu \mathrm{m}$ paraffin sections were cut and mounted onto glass slides. 3 sections per head were imaged at the same anatomical position and the retinal width and vacuolization was quantified using image $\mathrm{J}$ software. Graphpad 6 was used to determine statistical significance.

Drosophila Immunoblotting. Immunoblotting was performed as previously described ${ }^{\text {ss }}$. Briefly, TDP-43 or LacZ was expressed in the eye with gmr-GAL4, protein was extracted from 5-10 male (TDP-43) or female 
(LacZ) heads in $10 \mu \mathrm{l} / \mathrm{head}$ of $2 \mathrm{X}$ Laemelli buffer with $5 \%(\mathrm{v} / \mathrm{v}) \beta$-mercaptoethanol, denatured at $95^{\circ} \mathrm{C}$, chilled on ice for $5 \mathrm{~min}$ and centrifuged at $5000 \mathrm{rpm}$ for $5 \mathrm{~min}$ at $4^{\circ} \mathrm{C}$. Half a fly head $(5 \mu \mathrm{l})$ was electrophoresed on a 4\%-12\% bis-tris gel and transferred onto nitrocellulose by wet transfer (30 V for 65 mins). Blots were blocked in 5\% milk in TBST (TBS supplemented with 0.05\% TWEEN-20, pH 8). Primary antibodies made up in TBST were: TDP-43 (1 in 10,000; Proteintech, \#10782-2AP), $\alpha$-TubulinHRP (1 in 5,000; Cell Signaling Technology, \#9099) and $\beta$-galactosidase (1 in 15,000; Promega, \#Z3781). Horseradish peroxidase (HRP)-coupled secondary antibodies made up in TBST were goat anti-rabbit-HRP (1 in 5,000; EMD Millipore \#AP307P) and goat anti-mouse-HRP (1 in 10,000; abcam, ab6789). All experiments were carried out on three or more biological replicates, blots were quantified with Image Jv and statistical analysis was carried out using Graphpad prism 6 software.

Cell Culture. SH-SY5Y cells were obtained from the American Type Culture Collection (ATCC; CCL$2266^{\mathrm{TM}}$ ). BioID stable cell lines for were generated using retroviral transduction. HEK293 Phoenix cells (National Gene Vector Biorepository, Indianapolis, IN) were transfected with each construct using Lipofectamine 3000 (Thermo Fisher Scientific) per manufacturer's recommendation. The transfected cells were incubated at $37^{\circ} \mathrm{C}$ for $6 \mathrm{~h}$. After $6 \mathrm{~h}$ incubation, the transfected cells were replenished with fresh medium and further incubated at $32^{\circ} \mathrm{C}$ for $72 \mathrm{~h}$. The culture media was filtered through a $0.45-\mu \mathrm{m}$ filter and added to SH-SY5Y cells along with Polybrene (4 $\mu \mathrm{g} / \mathrm{ml}$; Santa Cruz Biotechnology, Dallas, TX). At $72 \mathrm{~h}$ after transduction, puromycin $(2.5 \mu \mathrm{g} / \mathrm{ml}$; Thermo Fisher Scientific) was added to the target cells. Stable cells lines were verified for fusion-protein expression and proper localization using IF and WB. The stable cell lines were maintained in $5.0 \% \mathrm{CO}_{2}$ at $37^{\circ} \mathrm{C}$ in DMEM/F12 1:1 (HyClone, Logan, UT) supplemented with $10 \%$ fetal bovine serum (FBS). All cells were tested monthly for mycoplasma contamination.

Immunofluorescence. Cells grown on glass coverslips were fixed in $3 \%$ (wt/vol) paraformaldehyde/phosphate-buffered saline (PBS) for $10 \mathrm{~min}$ and permeabilized by $0.4 \%$ (wt/vol) Triton X-100/PBS for $15 \mathrm{~min}$. For labeling fusion proteins, a chicken anti-BioID2 antibody was used (1:5000; BID2-CP-100; BioFront Technologies). The primary antibody was detected using Alexa Fluor 568- 
conjugated goat anti-chicken (1:1000; A11041; Thermo Fisher Scientific). Alexa Fluor 488-conjugated streptavidin (S32354; Thermo Fisher Scientific) was used to detect biotinylated proteins. DNA was detected with Hoechst dye 33342. Coverslips were mounted using 10\% (wt/vol) Mowiol 4-88 (Polysciences). Confocal images were obtained using a Nikon A1 confocal microscope $(60 \times / 1.49$ oil APO TIRF Nikon objective) with a charge-coupled device camera (CoolSnap HQ; Photometrics) linked to a workstation running NIS-Elements software (Nikon, Melville, NY). Epifluorescence images were captured using a Nikon Eclipse NiE $(20 \times / 0.75$ Plan Apo Nikon objective $)$ microscope.

Western Blot Analysis. To analyze total cell lysates by immunoblot, $1.2 \times 10^{6}$ cells were lysed in SDSPAGE sample buffer, boiled for $5 \mathrm{~min}$, and sonicated to shear DNA. Proteins were separated on 4-20\% gradient gels (Mini-PROTEAN TGX; Bio-Rad, Hercules, CA) and transferred to nitrocellulose membrane (Bio-Rad). After blocking with 10\% (vol/vol) adult bovine serum and $0.2 \%$ Triton X-100 in PBS for 30 min, the membrane was incubated with chicken anti-BioID2 antibody (1:5000; BID2-CP-100; BioFront Technologies) overnight, washed with PBS and detected using horseradish peroxidase (HRP)-conjugated anti-chicken (1:40,000; A9046; Sigma-Aldrich). The signals from antibodies were detected using enhanced chemiluminescence via a Bio-Rad ChemiDoc MP System (Bio-Rad, Hercules, CA). Following detection of BioID2, the membrane was quenched with $30 \% \mathrm{H}_{2} \mathrm{O}_{2}$ for $30 \mathrm{~min}$. To detect biotinylated proteins, the membrane was incubated with HRP-conjugated streptavidin $(1: 40,000$; ab7403; Abcam) in $0.4 \%$ Triton X100 in PBS for 45 min.

BioID Pulldowns. Large-scale BioID pulldowns were performed as described in $^{56}$ with four $10 \mathrm{~cm}$ dishes per sample instead of two. In brief, four $10 \mathrm{~cm}$ dishes at $80 \%$ confluency were incubated with $50 \mu \mathrm{M}$ biotin for 18 hours. Cells were lysed in $8 \mathrm{M}$ urea 50mM Tris $\mathrm{pH} 7.4$ containing protease inhibitor $(87785$, Thermo Fisher Scientific) and DTT, incubated with universal nuclease (88700, Thermo Fisher Scientific), and sonicated to further shear DNA. Lysates were precleared with Gelatin Sepharose 4B beads (17095601; GE Healthcare) for $2 \mathrm{~h}$ and then incubated with Streptavidin Sepharose High Performance beads (17511301, 
GE Healthcare) overnight. Streptavidin beads were washed four times with $8 \mathrm{M}$ urea 50mM Tris pH 7.4 wash buffer and resuspended in $50 \mathrm{mM}$ ammonium bicarbonate with $1 \mathrm{mM}$ biotin.

Sample preparation for mass spectrometry. Beads were resuspended with $8 \mathrm{M}$ urea, $50 \mathrm{mM}$ ammonium bicarbonate, and cysteine disulfide bonds were reduced with $10 \mathrm{mM}$ tris(2-carboxyethyl)phosphine (TCEP) at $30^{\circ} \mathrm{C}$ for $60 \mathrm{~min}$ and cysteines were then alkylated with $30 \mathrm{mM}$ iodoacetamide (IAA) in the dark at room temperature for $30 \mathrm{~min}$. Following alkylation, urea was diluted to $1 \mathrm{M}$ urea, and proteins were subjected to overnight digestion with mass spec grade Trypsin/Lys-C mix (Promega, Madison, WI). Finally, beads were pulled down and the solution with peptides collected into a new tube. Affinity purification was carried out in a Bravo AssayMap platform (Agilent) using AssayMap streptavidin cartridges (Agilent). Digested peptides were then desalted in a Bravo AssayMap platform (Agilent) using AssayMap C18 cartridges and dried down in a SpeedVac concentrator.

LC-MS/MS analysis. Prior to LC-MS/MS analysis, dried peptides were reconstituted with $2 \%$ ACN, $0.1 \%$ FA and concentration was determined using a NanoDrop ${ }^{\mathrm{TM}}$ spectrophometer (ThermoFisher). Samples were then analyzed by LC-MS/MS using a Proxeon EASY-nanoLC system (ThermoFisher) coupled to a Q-Exactive Plus mass spectrometer (Thermo Fisher Scientific). Peptides were separated using an analytical C18 Aurora column $(75 \mu \mathrm{m}$ x $250 \mathrm{~mm}, 1.6 \mu \mathrm{m}$ particles; IonOpticks $)$ at a flow rate of $300 \mathrm{~nL} / \mathrm{min}\left(60^{\circ} \mathrm{C}\right)$ using a 120 -min gradient: $1 \%$ to $5 \% \mathrm{~B}$ in $1 \mathrm{~min}, 6 \%$ to $23 \% \mathrm{~B}$ in $72 \mathrm{~min}, 23 \%$ to $34 \% \mathrm{~B}$ in $45 \mathrm{~min}$, and $34 \%$ to $48 \%$ B in $2 \min (\mathrm{A}=\mathrm{FA} 0.1 \%$; $\mathrm{B}=80 \% \mathrm{ACN}: 0.1 \% \mathrm{FA})$. The mass spectrometer was operated in positive data-dependent acquisition mode. MS1 spectra were measured in the Orbitrap in a mass-to-charge $(\mathrm{m} / \mathrm{z})$ of $350-1700$ with a resolution of 70,000 at $\mathrm{m} / \mathrm{z} 400$. Automatic gain control target was set to $1 \times 10^{6}$ with a maximum injection time of $100 \mathrm{~ms}$. Up to $12 \mathrm{MS} 2$ spectra per duty cycle were triggered, fragmented by $\mathrm{HCD}$, and acquired with a resolution of 17,500 and an AGC target of $5 \times 10^{4}$, an isolation window of $1.6 \mathrm{~m} / \mathrm{z}$ and a normalized collision energy of 25 . The dynamic exclusion was set to 20 seconds with a 10 ppm mass tolerance around the precursor. 
MS Data Analysis. All mass spectra were analyzed with MaxQuant software version 1.6.11.0. MS/MS spectra were searched against the Homo sapiens Uniprot protein sequence database (downloaded in January 2020) and GPM cRAP sequences (commonly known protein contaminants). Precursor mass tolerance was set to $20 \mathrm{ppm}$ and $4.5 \mathrm{ppm}$ for the first search where initial mass recalibration was completed and for the main search, respectively. Product ions were searched with a mass tolerance 0.5 Da. The maximum precursor ion charge state used for searching was 7. Carbamidomethylation of cysteine was searched as a fixed modification, while oxidation of methionine and acetylation of protein $\mathrm{N}$-terminal were searched as variable modifications. Enzyme was set to trypsin in a specific mode and a maximum of two missed cleavages was allowed for searching. The target-decoy-based false discovery rate (FDR) filter for spectrum and protein identification was set to $1 \%$. Interaction candidates were those proteins enriched at least $3 \mathrm{x}$ over control samples (BioID2-only) and identified in at least two of the three experimental triplicate samples $(\mathrm{N}>2)$.

Immunohistochemistry. Samples from the frontal cortex and spinal cord of ALS and control patients were obtained from the University of Michigan Brain Bank (Table 1). Consent for autopsy was obtained in accordance with guidelines from the University of Michigan Brain Bank who reviewed and confirmed that protocols met the the criteria for human-subjects research.

Table 1: Description of human samples obtained from the University of Michigan Brain Bank.

\begin{tabular}{|l|l|l|l|l|}
\hline Age at death & Sex & PMI (h) & Diagnosis & Section \\
\hline 45 & M & 48 & ALS & Frontal cortex \\
\hline 74 & F & 6 & Control & Frontal cortex \\
\hline 87 & M & 9 & Control & Frontal cortex \\
\hline 60 & F & 33 & ALS & Frontal cortex \\
\hline
\end{tabular}


Immunostaining was accomplished using the Dako Autostainer Link 48 (Agilent, USA). Anti-HspA5 antibody (Abcam ab21685) was used at 1:1000 with the Dako High $\mathrm{pH}$ Target Retrieval Solution (Tris/EDTA, pH 9; Agilent, USA) (20mins, 97 degrees) and the Dako Envision Flex Plus Mouse Link Kit (Agilent, USA) to detect the antibody along with the Dako DAB (Agilent, USA).

\section{Acknowledgements}

This work was supported by grants from the Center for Innovation in Brain Science (CIBS). Academy of Medical Sciences Springboard Fellowship (LM), University of Dundee start-up grant (LM), and National Institutes of Health (R35GM126949 to KJR; R01NS097542 and R01NS113943 to SJB; and P30AG053760 to the University of Michigan Brain Bank and Alzheimer's Disease Research Center). We also would like to acknowledge Mr. Matthew D. Perkins who assisted with postmortem tissue from the University of Michigan Brain Bank. Immunohistochemistry was performed at the Rogel Cancer Center Tissue and Molecular Pathology Shared Resource Laboratory at the University of Michigan (NIH P30 CA04659229).

The Imaging Core and Biochemistry Core at Sanford Research, which facilitated these studies, are supported by Institutional Development Awards from the National Institute of General Medical Sciences and the National Institutes of Health under Grant P20GM103620.

\section{Conflict of Interest}

The funders had no role in the design of the study; in the collection, analyses, or interpretation of data; in the writing of the manuscript, or in the decision to publish the results. Sanford Research has licensed BioID reagents to BioFront Technologies.

(1) Powers, E. T.; Morimoto, R. I.; Dillin, A.; Kelly, J. W.; Balch, W. E. Biological and Chemical Approaches to Diseases of Proteostasis Deficiency. Annual Review of Biochemistry. 2009. 
(2) Mackenzie, I. R. A.; Neumann, M. Molecular Neuropathology of Frontotemporal Dementia: Insights into Disease Mechanisms from Postmortem Studies. Journal of Neurochemistry. 2016.

(3) Taylor, J. P.; Brown, R. H.; Cleveland, D. W. Decoding ALS: From Genes to Mechanism. Nature. 2016, pp 197-206.

(4) Amador-Ortiz, C.; Lin, W. L.; Ahmed, Z.; Personett, D.; Davies, P.; Duara, R.; Graff-Radford, N. R.; Hutton, M. L.; Dickson, D. W. TDP-43 Immunoreactivity in Hippocampal Sclerosis and Alzheimer's Disease. Ann. Neurol. 2007, 61, 435-445.

(5) Nelson, P. T.; Dickson, D. W.; Trojanowski, J. Q.; Jack, C. R.; Boyle, P. A.; Arfanakis, K.; Rademakers, R.; Alafuzoff, I.; Attems, J.; Brayne, C.; et al. Limbic-Predominant Age-Related TDP43 Encephalopathy (LATE): Consensus Working Group Report. Brain 2019, 142, 1503-1527.

(6) Neumann, M.; Sampathu, D. M.; Kwong, L. K.; Truax, A. C.; Micsenyi, M. C.; Chou, T. T.; Bruce, J.; Schuck, T.; Grossman, M.; Clark, C. M.; et al. Ubiquitinated TDP-43 in Frontotemporal Lobar Degeneration and Amyotrophic Lateral Sclerosis. Science (80-. ). 2006, 314, 130-133.

(7) Walker, A. K.; Daniels, C. M. P. La; Goldman, J. E.; Trojanowski, J. Q.; Lee, V. M. Y.; Messing, A. Astrocytic TDP-43 Pathology in Alexander Disease. J. Neurosci. 2014, . https://doi.org/10.1523/JNEUROSCI.0248-14.2014.

(8) Scotter, E. L.; Vance, C.; Nishimura, A. L.; Lee, Y. B.; Chen, H. J.; Urwin, H.; Sardone, V.; Mitchell, J. C.; Rogelj, B.; Rubinsztein, D. C.; et al. Differential Roles of the Ubiquitin Proteasome System and Autophagy in the Clearance of Soluble and Aggregated TDP-43 Species. J. Cell Sci. 2014, $127,1263-1278$.

(9) Cascella, R.; Fani, G.; Bigi, A.; Chiti, F.; Cecchi, C. Partial Failure of Proteostasis Systems Counteracting TDP-43 Aggregates in Neurodegenerative Diseases. Int. J. Mol. Sci. 2019, 20.

(10) Liu, G.; Coyne, A. N.; Pei, F.; Vaughan, S.; Chaung, M.; Zarnescu, D. C.; Buchan, J. R. Endocytosis Regulates TDP-43 Toxicity and Turnover. Nat. Commun. 2017, 8.

(11) Shi, Y.; Lin, S.; Staats, K. A.; Li, Y.; Chang, W. H.; Hung, S. T.; Hendricks, E.; Linares, G. R.; Wang, Y.; Son, E. Y.; et al. Haploinsufficiency Leads to Neurodegeneration in C9ORF72 ALS/FTD 
Human Induced Motor Neurons. Nat. Med. 2018, 24.

(12) Leibiger, C.; Deisel, J.; Aufschnaiter, A.; Ambros, S.; Tereshchenko, M.; Verheijen, B. M.; Büttner, S.; Braun, R. J. Endolysosomal Pathway Activity Protects Cells from Neurotoxic TDP-43. Microbial Cell. 2018.

(13) François-Moutal, L.; Perez-Miller, S.; Scott, D. D.; Miranda, V. G.; Mollasalehi, N.; Khanna, M. Structural Insights Into TDP-43 and Effects of Post-Translational Modifications. Frontiers in Molecular Neuroscience. 2019.

(14) Prasad, A.; Bharathi, V.; Sivalingam, V.; Girdhar, A.; Patel, B. K. Molecular Mechanisms of TDP43 Misfolding and Pathology in Amyotrophic Lateral Sclerosis. Front. Mol. Neurosci. 2019, 12.

(15) Kim, G.; Gautier, O.; Tassoni-Tsuchida, E.; Ma, X. R.; Gitler, A. D. ALS Genetics: Gains, Losses, and Implications for Future Therapies. Neuron. 2020.

(16) Suk, T. R.; Rousseaux, M. W. C. The Role of TDP-43 Mislocalization in Amyotrophic Lateral Sclerosis. Molecular Neurodegeneration. 2020.

(17) Nitika; Porter, C. M.; Truman, A. W.; Truttmann, M. C. Post-Translational Modifications of Hsp70 Family Proteins: Expanding the Chaperone Code. Journal of Biological Chemistry. 2020.

(18) Clerico, E. M.; Pozhidaeva, A. K.; Jansen, R. M.; Özden, C.; Tilitsky, J. M.; Gierasch, L. M. Selective Promiscuity in the Binding of E. Coli Hsp70 to an Unfolded Protein. Proc. Natl. Acad. Sci. 2021, 118.

(19) Radons, J. The Human HSP70 Family of Chaperones: Where Do We Stand? Cell Stress and Chaperones. 2016.

(20) Ambrose, A. J.; Chapman, E. Function, Therapeutic Potential, and Inhibition of Hsp70 Chaperones. Journal of Medicinal Chemistry. 2021.

(21) Clerico, E. M.; Tilitsky, J. M.; Meng, W.; Gierasch, L. M. How Hsp70 Molecular Machines Interact with Their Substrates to Mediate Diverse Physiological Functions. Journal of Molecular Biology. 2015, pp 1575-1588.

(22) Kim, H. J.; Joo, H. J.; Kim, Y. H.; Ahn, S.; Chang, J.; Hwang, K. B.; Lee, D. H.; Lee, K. J. Systemic 
Analysis of Heat Shock Response Induced by Heat Shock and a Proteasome Inhibitor MG132. PLoS One 2011, 6 .

(23) Rosenzweig, R.; Nillegoda, N. B.; Mayer, M. P.; Bukau, B. The Hsp70 Chaperone Network. Nature Reviews Molecular Cell Biology. 2019.

(24) Inda, M. C.; Joshi, S.; Wang, T.; Bolaender, A.; Gandu, S.; Koren, J.; Che, A. Y.; Taldone, T.; Yan, P.; Sun, W.; et al. The Epichaperome Is a Mediator of Toxic Hippocampal Stress and Leads to Protein Connectivity-Based Dysfunction. Nat. Commun. 2020, 11.

(25) Rodina, A.; Wang, T.; Yan, P.; Gomes, E. D.; Dunphy, M. P. S.; Pillarsetty, N.; Koren, J.; Gerecitano, J. F.; Taldone, T.; Zong, H.; et al. The Epichaperome Is an Integrated Chaperome Network That Facilitates Tumour Survival. Nature 2016, 538.

(26) Batulan, Z.; Shinder, G. A.; Minotti, S.; He, B. P.; Doroudchi, M. M.; Nalbantoglu, J.; Strong, M. J.; Durham, H. D. High Threshold for Induction of the Stress Response in Motor Neurons Is Associated with Failure to Activate HSF1. J. Neurosci. 2003, 23, 5789-5798.

Tidwell, J. L.; Houenou, L. J.; Tytell, M. Administration of Hsp70 in Vivo Inhibits Motor and Sensory Neuron Degeneration. Cell Stress Chaperones 2004, 9, 88-98.

(28) Kitamura, A.; Iwasaki, N.; Kinjo, M. Molecular Chaperone HSP70 Prevents Formation of Inclusion Bodies of the 25-KDa C-Terminal Fragment of TDP-43 by Preventing Aggregate Accumulation. Cell Stress Chaperones 2018, 23, 1177-1183.

(29) Gifondorwa, D. J.; Robinson, M. B.; Hayes, C. D.; Taylor, A. R.; Prevette, D. M.; Oppenheim, R. W.; Caress, J.; Milligan, C. E. Exogenous Delivery of Heat Shock Protein 70 Increases Lifespan in a Mouse Model of Amyotrophic Lateral Sclerosis. J. Neurosci. 2007, 27, 13173-13180.

(30) Deane, C. A. S.; Brown, I. R. Induction of Heat Shock Proteins in Differentiated Human Neuronal Cells Following Co-Application of Celastrol and Arimoclomol. Cell Stress Chaperones 2016, 21.

(31) Freibaum, B. D.; Chitta, R. K.; High, A. A.; Taylor, J. P. Global Analysis of TDP-43 Interacting Proteins Reveals Strong Association with RNA Splicing and Translation Machinery. J. Proteome Res. 2010, 9, 1104-1120. 
(32) Udan-Johns, M.; Bengoechea, R.; Bell, S.; Shao, J.; Diamond, M. I.; True, H. L.; Weihl, C. C.;

Baloh, R. H. Prion-like Nuclear Aggregation of TDP-43 during Heat Shock Is Regulated by HSP40/70 Chaperones. Hum. Mol. Genet. 2014, 23, 157-170.

(33) Yu, H.; Lu, S.; Gasior, K.; Singh, D.; Vazquez-Sanchez, S.; Tapia, O.; Toprani, D.; Beccari, M. S.;

Yates, J. R.; Da Cruz, S.; et al. HSP70 Chaperones RNA-Free TDP-43 into Anisotropic Intranuclear Liquid Spherical Shells. Science 2020, . https://doi.org/10.1126/science.abb4309.

(34) Roux, K. J.; Kim, D. I.; Burke, B.; May, D. G. BioID: A Screen for Protein-Protein Interactions. Curr. Protoc. Protein Sci. 2018, 91.

(35) Elefant, F.; Palter, K. B. Tissue-Specific Expression of Dominant Negative Mutant Drosophila HSC70 Causes Developmental Defects and Lethality. Mol. Biol. Cell 1999, . https://doi.org/10.1091/mbc.10.7.2101.

(36) Imamoto, N.; Matsuoka, Y.; Kurihara, T.; Kohno, K.; Miyagi, M.; Sakiyama, F.; Okada, Y.; Tsunasawa, S.; Yoneda, Y. Antibodies against 70-KD Heat Shock Cognate Protein Inhibit Mediated Nuclear Import of Karyophilic Proteins. J. Cell Biol. 1992, 119.

Fu, J.; Wei, C.; He, J.; Zhang, L.; Zhou, J.; Balaji, K. S.; Shen, S.; Peng, J.; Sharma, A.; Fu, J. Evaluation and Characterization of HSPA5 (GRP78) Expression Profiles in Normal Individuals and Cancer Patients with COVID-19. Int. J. Biol. Sci. 2021, 17.

(38) Wu, Y.; Guo, Z.; Liu, F.; Yao, K.; Gao, M.; Luo, Y.; Zhang, Y. Sp110 Enhances Macrophage Resistance to Mycobacterium Tuberculosis via Inducing Endoplasmic Reticulum Stress and Inhibiting Anti-Apoptotic Factors. Oncotarget 2017, 8.

(39) Shi, W.; Xu, G.; Wang, C.; Sperber, S. M.; Chen, Y.; Zhou, Q.; Deng, Y.; Zhao, H. Heat Shock 70KDa Protein 5 (Hspa5) Is Essential for Pronephros Formation by Mediating Retinoic Acid Signaling. J. Biol. Chem. 2015, 290.

(40) Van Durme, J.; Maurer-Stroh, S.; Gallardo, R.; Wilkinson, H.; Rousseau, F.; Schymkowitz, J. Accurate Prediction of DnaK-Peptide Binding via Homology Modelling and Experimental Data. PLoS Comput. Biol. 2009, 5. 
(41) Dang, M.; Kang, J.; Lim, L.; Li, Y.; Wang, L.; Song, J. ATP Is a Cryptic Binder of TDP-43 RRM Domains to Enhance Stability and Inhibit ALS/AD-Associated Fibrillation. Biochem. Biophys. Res. Commun. 2020, . https://doi.org/10.1016/j.bbrc.2019.11.088.

(42) Lukavsky, P. J.; Daujotyte, D.; Tollervey, J. R.; Ule, J.; Stuani, C.; Buratti, E.; Baralle, F. E.; Damberger, F. F.; Allain, F. H. T. Molecular Basis of UG-Rich RNA Recognition by the Human Splicing Factor TDP-43. Nat. Struct. Mol. Biol. 2013, 20, 1443-1449.

(43) Bhardwaj, A.; Myers, M. P.; Buratti, E.; Baralle, F. E. Characterizing TDP-43 Interaction with Its RNA Targets. Nucleic Acids Res. 2013, 41, 5062-5074.

(44) Ederle, H.; Funk, C.; Abou-Ajram, C.; Hutten, S.; Funk, E. B. E.; Kehlenbach, R. H.; Bailer, S. M.; Dormann, D. Nuclear Egress of TDP-43 and FUS Occurs Independently of Exportin-1/CRM1. Sci. Rep. 2018, 8 .

(45) Pinarbasi, E. S.; Cağatay, T.; Fung, H. Y. J.; Li, Y. C.; Chook, Y. M.; Thomas, P. J. Active Nuclear Import and Passive Nuclear Export Are the Primary Determinants of TDP-43 Localization. Sci. Rep. 2018, $8,10.1038 / \mathrm{s} 41598-018-25008-4$.

(46) Saini, A.; Chauhan, V. S. Delineation of the Core Aggregation Sequences of TDP-43 C-Terminal Fragment. ChemBioChem 2011, . https://doi.org/10.1002/cbic.201100427.

(47) Shodai, A.; Ido, A.; Fujiwara, N.; Ayaki, T.; Morimura, T.; Oono, M.; Uchida, T.; Takahashi, R.; Ito, H.; Urushitani, M. Conserved Acidic Amino Acid Residues in a Second RNA Recognition Motif Regulate Assembly and Function of TDP-43. PLoS One 2012, 7.

(48) Elden, A. C.; Kim, H. J.; Hart, M. P.; Chen-Plotkin, A. S.; Johnson, B. S.; Fang, X.; Armakola, M.; Geser, F.; Greene, R.; Lu, M. M.; et al. Ataxin-2 Intermediate-Length Polyglutamine Expansions Are Associated with Increased Risk for ALS. Nature 2010, 466, 1069-1075.

(49) Kim, H. J.; Raphael, A. R.; Ladow, E. S.; Mcgurk, L.; Weber, R. A.; Trojanowski, J. Q.; Lee, V. M. Y.; Finkbeiner, S.; Gitler, A. D.; Bonini, N. M. Therapeutic Modulation of EIF2 $\alpha$ Phosphorylation Rescues TDP-43 Toxicity in Amyotrophic Lateral Sclerosis Disease Models. Nat. Genet. 2014, . https://doi.org/10.1038/ng.2853. 
(50) Vígh, L.; Literáti, P. N.; Horváth, I.; Török, Z.; Balogh, G.; Glatz, A.; Kovács, E.; Boros, I.; Ferdinándy, P.; Farkas, B.; et al. Bimoclomol: A Nontoxic, Hydroxylamine Derivative with Stress Protein- Inducing Activity and Cytoprotective Effects. Nat. Med. 1997, . https://doi.org/10.1038/nm1097-1150.

(51) Berson, A.; Goodman, L. D.; Sartoris, A. N.; Otte, C. G.; Aykit, J. A.; Lee, V. M. Y.; Trojanowski, J. Q.; Bonini, N. M. Drosophila Refl/ALYREF Regulates Transcription and Toxicity Associated with ALS/FTD Disease Etiologies. Acta Neuropathol. Commun. 2019, . https://doi.org/10.1186/s40478-019-0710-x.

(52) Coyne, A. N.; Lorenzini, I.; Chou, C. C.; Torvund, M.; Rogers, R. S.; Starr, A.; Zaepfel, B. L.; Levy, J.; Johannesmeyer, J.; Schwartz, J. C.; et al. Post-Transcriptional Inhibition of Hsc70-4/HSPA8 Expression Leads to Synaptic Vesicle Cycling Defects in Multiple Models of ALS. Cell Rep. 2017, . https://doi.org/10.1016/j.celrep.2017.09.028.

(53) Armakola, M.; Higgins, M. J.; Figley, M. D.; Barmada, S. J.; Scarborough, E. A.; Diaz, Z.; Fang, X.; Shorter, J.; Krogan, N. J.; Finkbeiner, S.; et al. Inhibition of RNA Lariat Debranching Enzyme Suppresses TDP-43 Toxicity in ALS Disease Models. Nat. Genet. 2012, . https://doi.org/10.1038/ng.2434.

(54) Leibiger, C.; Deisel, J.; Aufschnaiter, A.; Ambros, S.; Tereshchenko, M.; Verheijen, B. M.; Büttner, S.; Braun, R. J. TDP-43 Controls Lysosomal Pathways Thereby Determining Its Own Clearance and Cytotoxicity. Hum. Mol. Genet. 2018, . https://doi.org/10.1093/hmg/ddy066.

(55) McGurk, L.; Gomes, E.; Guo, L.; Mojsilovic-Petrovic, J.; Tran, V.; Kalb, R. G.; Shorter, J.; Bonini, N. M. Poly(ADP-Ribose) Prevents Pathological Phase Separation of TDP-43 by Promoting Liquid Demixing and Stress Granule Localization. Mol. Cell 2018, 71, 703-717.e9.

(56) Pons, M.; Prieto, S.; Miguel, L.; Frebourg, T.; Campion, D.; Suñé, C.; Lecourtois, M. Identification of TCERG1 as a New Genetic Modulator of TDP-43 Production in Drosophila. Acta Neuropathol. Commun. 2018, . https://doi.org/10.1186/s40478-018-0639-5.

(57) Chou, C. C.; Zhang, Y.; Umoh, M. E.; Vaughan, S. W.; Lorenzini, I.; Liu, F.; Sayegh, M.; Donlin- 
Asp, P. G.; Chen, Y. H.; Duong, D. M.; et al. TDP-43 Pathology Disrupts Nuclear Pore Complexes and Nucleocytoplasmic Transport in ALS/FTD. Nat. Neurosci. 2018, . https://doi.org/10.1038/s41593-017-0047-3.

(58) Berson, A.; Sartoris, A.; Nativio, R.; Van Deerlin, V.; Toledo, J. B.; Porta, S.; Liu, S.; Chung, C. Y.; Garcia, B. A.; Lee, V. M. Y.; et al. TDP-43 Promotes Neurodegeneration by Impairing Chromatin Remodeling. Curr. Biol. 2017, . https://doi.org/10.1016/j.cub.2017.10.024.

(59) Sun, F. C.; Wei, S.; Li, C. W.; Chang, Y. S.; Chao, C. C.; Lai, Y. K. Localization of GRP78 to Mitochondria under the Unfolded Protein Response. Biochem. J. 2006, . https://doi.org/10.1042/BJ20051916.

(60) Zhang, Y.; Liu, R.; Ni, M.; Gill, P.; Lee, A. S. Cell Surface Relocalization of the Endoplasmic Reticulum Chaperone and Unfolded Protein Response Regulator GRP78/BiP. J. Biol. Chem. 2010, . https://doi.org/10.1074/jbc.M109.087445.

(61) Mandell, R. B.; Feldherr, C. M. Identification of Two HSP70-Related Xenopus Oocyte Proteins That Are Capable of Recycling across the Nuclear Envelope. J. Cell Biol. 1990, 111.

(62) Carrara, M.; Prischi, F.; Ali, M. M. U. UPR Signal Activation by Luminal Sensor Domains. International Journal of Molecular Sciences. 2013.

(63) Bertolotti, A.; Zhang, Y.; Hendershot, L. M.; Harding, H. P.; Ron, D. Dynamic Interaction of BiP and ER Stress Transducers in the Unfolded-Protein Response. Nat. Cell Biol. 2000, 2.

(64) Mann, J. R.; Gleixner, A. M.; Mauna, J. C.; Gomes, E.; DeChellis-Marks, M. R.; Needham, P. G.; Copley, K. E.; Hurtle, B.; Portz, B.; Pyles, N. J.; et al. RNA Binding Antagonizes Neurotoxic Phase Transitions of TDP-43. Neuron 2019, 102, 321-338.e8.

(65) Chen, H. J.; Topp, S. D.; Hui, H. S.; Zacco, E.; Katarya, M.; McLoughlin, C.; King, A.; Smith, B. N.; Troakes, C.; Pastore, A.; et al. RRM Adjacent TARDBP Mutations Disrupt RNA Binding and Enhance TDP-43 Proteinopathy. Brain 2019, 142.

(66) Jin, H.; Mimura, N.; Kashio, M.; Koseki, H.; Aoe, T. Late-Onset of Spinal Neurodegeneration in Knock-in Mice Expressing a Mutant BIP. PLoS One 2014, 9. 
(67) Filézac De L’Etang, A.; Maharjan, N.; Cordeiro Branã, M.; Ruegsegger, C.; Rehmann, R.; Goswami, A.; Roos, A.; Troost, D.; Schneider, B. L.; Weis, J.; et al. Marinesco-Sjögren Syndrome Protein SIL1 Regulates Motor Neuron Subtype-Selective ER Stress in ALS. Nat. Neurosci. 2015, 18.

(68) McGurk, L.; Rifai, O.; Bonini, N. M. TDP-43 a Protein Central to Amyotrophic Lateral Sclerosis Is Destabilized by Tankyrase-1/2. J. Cell Sci. 2020, . https://doi.org/10.1242/JCS.245811.

(69) Wang, J.; Lee, J.; Liem, D.; Ping, P. HSPA5 Gene Encoding Hsp70 Chaperone BiP in the Endoplasmic Reticulum. Gene. 2017.

(70) Wang, X.; Olberding, K. E.; White, C.; Li, C. Bcl-2 Proteins Regulate ER Membrane Permeability to Luminal Proteins during ER Stress-Induced Apoptosis. Cell Death Differ. 2011, . https://doi.org/10.1038/cdd.2010.68.

(71) Sasaki, S.; Takeda, T.; Shibata, N.; Kobayashi, M. Alterations in Subcellular Localization of TDP43 Immunoreactivity in the Anterior Horns in Sporadic Amyotrophic Lateral Sclerosis. Neurosci. Lett. 2010, 478 .

(72) Ito, Y.; Yamada, M.; Tanaka, H.; Aida, K.; Tsuruma, K.; Shimazawa, M.; Hozumi, I.; Inuzuka, T.; Takahashi, H.; Hara, H. Involvement of CHOP, an ER-Stress Apoptotic Mediator, in Both Human Sporadic ALS and ALS Model Mice. Neurobiol. Dis. 2009, . https://doi.org/10.1016/j.nbd.2009.08.013.

(73) Kiskinis, E.; Sandoe, J.; Williams, L. A.; Boulting, G. L.; Moccia, R.; Wainger, B. J.; Han, S.; Peng, T.; Thams, S.; Mikkilineni, S.; et al. Pathways Disrupted in Human ALS Motor Neurons Identified through Genetic Correction of Mutant SOD1. Cell Stem Cell 2014, . https://doi.org/10.1016/j.stem.2014.03.004.

(74) Montibeller, L.; de Belleroche, J. Amyotrophic Lateral Sclerosis (ALS) and Alzheimer's Disease (AD) Are Characterised by Differential Activation of ER Stress Pathways: Focus on UPR Target Genes. Cell Stress Chaperones 2018, . https://doi.org/10.1007/s12192-018-0897-y.

(75) Li, W. W.; Sistonen, L.; Morimoto, R. I.; Lee, A. S. Stress Induction of the Mammalian GRP78/BiP 
Protein Gene: In Vivo Genomic Footprinting and Identification of P70CORE from Human Nuclear Extract as a DNA-Binding Component Specific to the Stress Regulatory Element. Mol. Cell. Biol. $1994,14$.

(76) McMillan, D. R.; Xiao, X.; Shao, L.; Graves, K.; Benjamin, I. J. Targeted Disruption of Heat Shock Transcription Factor 1 Abolishes Thermotolerance and Protection against Heat-Inducible Apoptosis. J. Biol. Chem. 1998, 273.

(77) Hong, M.; Lin, M. Y.; Huang, J. M.; Baumeister, P.; Hakre, S.; Roy, A. L.; Lee, A. S. Transcriptional Regulation of the Grp78 Promoter by Endoplasmic Reticulum Stress: Role of TFII-I and Its Tyrosine Phosphorylation. J. Biol. Chem. 2005, 280.

(78) McGurk, L.; Rifai, O. M.; Shcherbakova, O.; Perlegos, A. E.; Byrns, C. N.; Carranza, F. R.; Zhou, H. W.; Kim, H.-J.; Zhu, Y.; Bonini, N. M. Toxicity of Pathogenic Ataxin-2 in Drosophila Shows Dependence on a Pure CAG Repeat Sequence . Hum. Mol. Genet. 2021, 30.

(79) Rueden, C. T.; Schindelin, J.; Hiner, M. C.; DeZonia, B. E.; Walter, A. E.; Arena, E. T.; Eliceiri, K. W. ImageJ2: ImageJ for the next Generation of Scientific Image Data. BMC Bioinformatics 2017, . https://doi.org/10.1186/s12859-017-1934-z. 


\section{Supplementary Files}

This is a list of supplementary files associated with this preprint. Click to download.

- SupplementaryinformationFINAL.pdf

- Tables1.xlsx 